\title{
Regulation of the Arf/p53 Tumor Surveillance Network by E2F
}

\author{
P.J. Iaquinta, A. Aslanian,* AND J.A. Lees \\ Center for Cancer Research, Massachusetts Institute of Technology, Cambridge, Massachusetts 02139
}

\begin{abstract}
Deregulation of the cell cycle machinery plays a critical role in tumorigenesis. In particular, functional inactivation of the retinoblastoma protein (pRB) is a key event. pRB's tumor suppressive activity is at least partially dependent on its ability to regulate the activity of the E2F transcription factors. E2F controls the expression of genes that encode the cellular proliferation machinery. E2F can also trigger apoptosis when it is inappropriately expressed. Here we present evidence that E2F acts to directly regulate the Arf/p53 tumor surveillance network. In normal cells, a single member of the E2F family, E2F3, participates in the transcriptional silencing of Arf. In response to oncogenic stress, the activating E2Fs, E2F1, 2, and E2F3A, all associate with $A r f$ and promote its transcription. These findings raise the possibility that E2F acts as a sensor of inappropriate versus normal proliferative signals and determines whether or not the Arf/p53 tumor surveillance network is engaged.
\end{abstract}

The retinoblastoma gene $(R B-1)$ was the first identified tumor suppressor, and it is mutated in one-third of all human tumors (Sherr 1996). In normal cells, the retinoblastoma protein (pRB) arrests cells in the $\mathrm{G}_{1}$ phase of the cell cycle. This inhibition is relieved through activation of the cell cycle kinase cyclin $\mathrm{D} / \mathrm{cdk} 4$ and the sequential phosphorylation of $\mathrm{pRB}$ by cyclin $\mathrm{D} / \mathrm{cdk} 4$ and cyclin E/cdk2. Negative growth signals promote cell cycle arrest by increasing the expression of the cdk inhibitor, p16, a known tumor suppressor. Most, if not all, human tumors carry either inactivating mutations in $R b$ or $p 16$ or activating mutations that up-regulate cyclin D/cdk4 (Sherr 1996). Thus, functional inactivation of $\mathrm{pRB}$ is an essential step in the tumorigenic process.

Molecular studies have identified numerous proteins that bind to the pRB tumor suppressor (Morris and Dyson 2001). The vast majority of these proteins are known components of transcriptional complexes including chromatin regulators (e.g., swi/snf proteins, HDACs, and histone methylases) and transcription factors. Of these interactors, the E2F transcription factors are by far the best characterized (Trimarchi and Lees 2002; Dimova and Dyson 2005). As we outline below, there is overwhelming evidence that pRB's tumor suppressive activity is at least partially dependent on its ability to regulate E2F. In normal cells, E2F controls the expression of genes that encode essential cellular proteins including cell cycle regulators, nucleotide biosynthesis enzymes, histone variants, DNA damage regulators, and DNA repair proteins (Trimarchi and Lees 2002; Cam and Dynlacht 2003). These classic E2F-responsive genes share a highly characteristic pattern of transcription: They are repressed during $\mathrm{G}_{0} / \mathrm{G}_{1}$, get activated in late $\mathrm{G}_{1}$, and are switched off again in $\mathrm{S} / \mathrm{G}_{2} / \mathrm{M}$. This periodic expression is controlled by the concerted action of the E2F proteins and their associated regulatory proteins.

We know that the endogenous E2F activity is generated from the concerted action of multiple E2F complexes (Tri-

*Present address: Salk Institute, La Jolla, California 92037. marchi and Lees 2002). Most E2F complexes are heterodimeric, containing one $\mathrm{E} 2 \mathrm{~F}$ and one DP protein, but recent studies have shown that E2F7 and E2F8 function as homodimers (Trimarchi and Lees 2002; de Bruin et al. 2003; Di Stefano et al. 2003; Christensen et al. 2005; Maiti et al. 2005). The biological properties of individual E2F complexes are determined largely by the E2F subunit. To date, eight $E 2 f$ genes and nine E2F proteins have been identified (Christensen et al. 2005; Dimova and Dyson 2005; Maiti et al. 2005). The discrepancy between these two numbers is explained by the fact that the $E 2 f 3$ gene encodes two distinct proteins, called E2F3A and E2F3B, through the use of different promoters and $5^{\prime}$ coding exons (Adams et al. 2000; He et al. 2000; Leone et al. 2000). The E2F proteins can be divided into different subgroups based on significant differences in their roles and upstream regulation (Fig. 1). The most striking distinction is that individual E2Fs appear to be involved predominantly in either the repression or activation of E2F-responsive genes.

As their names imply, E2F6, 7, and 8 were the latest E2F family members to be identified. They share significant sequence homology with the core DNA-binding domain of the other E2F proteins, and the overexpression of E2F6, 7, or 8 is sufficient to enforce the repression of classic E2F-responsive genes (Morkel et al. 1997; Cartwright et al. 1998; Trimarchi et al. 1998; de Bruin et al. 2003; Di Stefano et al. 2003; Christensen et al. 2005; Maiti et al. 2005). We still understand little about the role and regulation of these three E2Fs in vivo. Notably, in contrast to the other E2F family members, E2F6, 7, and 8 are not regulated by their association with $\mathrm{pRB}$ or the related pocket proteins, p107 and p130. Without even this circumstantial link, it remains an open question as to whether E2F6, 7, and/or 8 might influence tumorigenesis.

E2F4 and 5 constitute the second E2F subgroup (Trimarchi and Lees 2002; Dimova and Dyson 2005). These E2Fs are thought to play a key role in the transcriptional repression of classic E2F-responsive genes via recruitment of p130 or p107 and their associated histone deacetylases. The nuclear localization of E2F4 and 5 appears to be dependent on their association with the pocket 


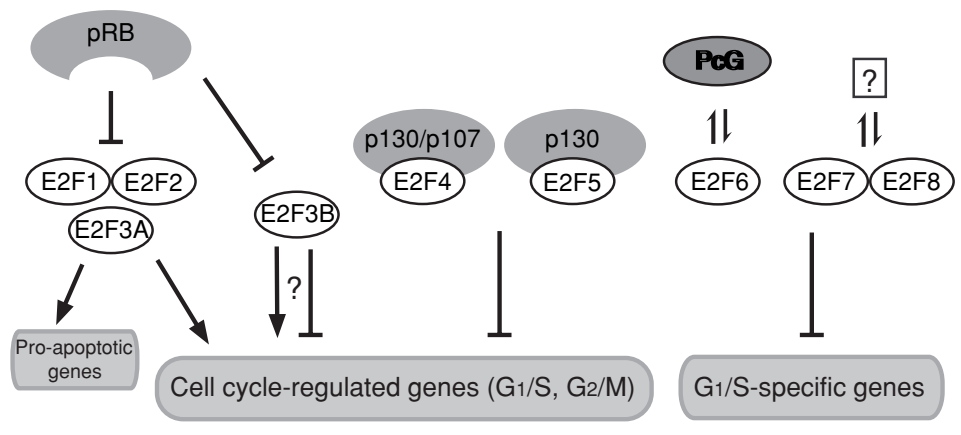

Figure 1. E2F family members are divided into different subgroups, with different functional properties. The different E2F subgroups positively or negatively regulate different subclasses of genes (shaded boxes), partially dependent on their upstream regulators and associated proteins (gray ovals). proteins (Verona et al. 1997; Gaubatz et al. 2001). Chromatin immunoprecipitation (ChIP) assays show that E2F4, p107, and p130 bind to classic E2F-responsive promoters in $G_{0} / G_{1}$ when these genes are actively repressed (Ren et al. 2002; Cam et al. 2004). Cells lacking E2F4 and 5 proliferate normally, but they have a defective growth-arrest response due to their inability to appropriately silence E2F-responsive genes (Gaubatz et al. 2000; Landsberg et al. 2003). More recently, E2F3B was cloned and shown to be expressed in quiescent cells in a similar manner to E2F4 and 5 (He et al. 2000; Leone et al. 2000). On the basis of this observation, it was proposed that E2F3B would cooperate with E2F4 and 5 in mediating the transcriptional repression of classic E2F-responsive genes (Leone et al. 2000).

The third E2F subgroup includes E2F1, 2, and 3A (Lees et al. 1993). These E2Fs are potent transcriptional activators, and they are thought to play an important role in the transcriptional activation of classic E2F-responsive genes. E2F1, 2, and 3A are specifically regulated by $\mathrm{pRB}$ and not by p107 or p130 in normal cells (Lees et al. 1993). The resulting $\mathrm{pRB} / \mathrm{E} 2 \mathrm{~F} 1,2$, or $3 \mathrm{~A}$ complexes are not detected at the promoters of classic E2F-responsive genes in $G_{0} / G_{1}$ cells (Ren et al. 2002; Cam et al. 2004), suggesting that they do not participate in their active repression. However, E2F1, 2, and 3A bind to these promoters in mid to late $G_{1}$, coincident with their release from $\mathrm{pRB}$ and the activation of these genes (Ren et al. 2002; Cam et al. 2004).

Overexpression studies show that the activating E2Fs can override growth-arrest signals and promote cellular proliferation (Johnson et al. 1994; Qin et al. 1994; Lukas et al. 1996). At the same time, these E2Fs are potent inducers of apoptosis (Qin et al. 1994; Wu and Levine 1994; Hiebert et al. 1995; Hsieh et al. 1997; Phillips et al. 1997, 1999). These opposing forces of proliferation and apoptosis are both classic hallmarks of tumor cells (Hanahan and Weinberg 2000). Mouse models have provided unequivocal proof that the activating E2Fs, E2fl and $E 2 f 3$, promote the development of pRB-deficient tumors (Pan et al. 1998; Yamasaki et al. 1998; McCaffrey et al. 1999; Ziebold et al. 2003; Denchi and Helin 2005). Moreover, either directly or indirectly, these E2Fs contribute to both the ectopic proliferation and apoptosis that occur within the developing tumors.

Considerable attention has focused on understanding the mechanism(s) by which the activating E2Fs stimulate apoptosis. To date, a variety of pro-apoptotic regulators have been identified as candidate E2F-responsive genes. The best-known example is the Arf tumor suppressor. The product of the Arf gene, called p19 ${ }^{\text {Arf }}$ (in mouse), is a key component of the $p 53$ tumor surveillance network. p19 Arf exists at low or undetectable levels in most normal cells but is activated by inappropriate proliferative signals (Sherr and Weber 2000). Once it is expressed, p19 Arf inhibits mdm2, a ubiquitin ligase, allowing activation of the p53 tumor suppressor and induction of p53-responsive genes. These target genes promote cell cycle arrest or apoptosis and thereby counteract the effect of the abnormal proliferative signals. Essentially, p19 ${ }^{\text {Arf }}$ acts as a defense against oncogenic signals. This explains why inactivation of the p19 Arf - mdm2-p53 network, through either inactivating mutations in $A r f$ or $p 53$ or amplification of $m d m 2 / h d m 2$, is critical for tumor development (Sherr 2001). Importantly, the analysis of a mouse strain that expresses GFP in place of $\mathrm{p} 19^{\mathrm{Arf}}$ provides direct in vivo evidence that Arf is not activated by normal proliferation but is reliably induced by the oncogenic signals that trigger tumorigenesis (Zindy et al. 2003).

Understanding the mechanism by which Arf responds to inappropriate, but not normal, proliferative signals remains a key goal. A variety of studies suggest a role for E2F in the activation of $\operatorname{Arf}$ (Phillips and Vousden 2001). The Arf promoter contains several consensus E2F-binding sites, and the ectopic expression of the activating E2Fs is sufficient to trigger Arf transcription (DeGregori et al. 1997; Phillips et al. 1999). However, it was unclear whether this regulation was direct because the identified E2F sites were dispensable for E2F-dependent Arf activation (Parisi et al. 2002; Berkovich et al. 2003) and many oncogenes lead to the activation of $\operatorname{Arf}$ (Sherr and Weber 2000). Moreover, Arf is not expressed in the cell-cycle-dependent manner that is typical of classic E2F-responsive genes. Thus, it was unclear whether Arf is a genuine E2F-responsive gene in vivo. As we describe below, our data show that distinct E2F family members contribute to the transcriptional regulation of Arf in normal versus tumor cells.

\section{MATERIALS AND METHODS}

MEF preparation and culture. $E 2 f 3^{+/-}$mice (Humbert et al. 2000) were intercrossed, and MEFs were prepared from E13.5 embryos as described previously (Humbert et al. 2000). For serum starvation and release experiments, 
passage- 4 MEFs were incubated in low serum $(0.1 \%$ FCS) for 72 hours, and subsequently fed with media containing $10 \%$ FCS. To monitor DNA synthesis, cells were labeled with $5 \mu \mathrm{Ci}\left[{ }^{3} \mathrm{H}\right]$ thymidine for 1 hour and harvested, and then $\left[{ }^{3} \mathrm{H}\right]$ thymidine incorporation was measured as described previously (Moberg et al. 1996).

Protein preparation and western blotting. Passage-4 MEFs were plated onto $15-\mathrm{cm}$ dishes at $3 \times 10^{6} \mathrm{cells} / \mathrm{dish}$, and cell cycle reentry was performed as described above. For each time point, cells were harvested, and total protein was isolated as described previously (Moberg et al. 1996). Western blotting was performed using $100 \mu \mathrm{g}$ of wholecell extract and antibodies specific for cyclin A (Santa Cruz sc-596), p19 ${ }^{\text {Arf }}$ (Novus NB200-106), p21 Cip1 (Santa Cruz sc-6246), phospho-p53 (Ser15) (Cell Signaling Technology \#9284), or $\beta$-Tubulin (TUB2.1, Sigma T4026).

Chromatin immunoprecipitation (ChIP). ChIP was performed essentially as described previously (Takahashi et al. 2000; Aslanian et al. 2004). Sonicated, cross-linked chromatin corresponding to approximately $3 \times 10^{6}$ cells was immunoprecipitated with the following antibodies: normal rabbit IgG (control), sc-2027; E2F1, sc-193; E2F2, sc-633x; E2F3A, sc-879x; E2F3A+B, sc-878x; E2F4, sc-1082x; p130, sc-317x (all from Santa Cruz Biotechnology). $3-4 \%$ of the precipitated DNA, or $0.5 \%$ of input DNA, was amplified by 30 cycles of PCR using primer sequences for Arf, p107, or $1 \mathrm{~kb}$ upstream of the E2f1 promoter (Aslanian et al. 2004). PCR products were stained with ethidium bromide after resolution on $8 \%$ polyacrylamide gels.

Retroviral infection. Infections were performed exactly as described previously (Serrano et al. 1996). Wildtype MEFs were infected and subsequently selected for 2 days in $2 \mathrm{mg} / \mathrm{ml}$ puromycin, grown for an additional 2 days, and then subjected to ChIP analysis as described below. pBabe-Puro and LPC-12S-E1A have been described previously (de Stanchina et al. 1998).

\section{RESULTS}

\section{E2f3 Is Required for Arf Repression in Unstressed Cells}

Our first insight into the role of E2F in the regulation of Arf came from the analysis of mouse embryonic fibroblasts (MEFs) derived from an E2f3 mutant mouse strain. This strain lacks the sequences encoding the E2F DNAbinding domain and consequently is deficient for both the E2F3A and E2F3B proteins (Humbert et al. 2000). The $E 2 f 3^{-1-}$ MEFs have a reduced proliferative capacity and also a dramatic impairment in their ability to reenter the cell cycle in response to growth factor stimulation (Humbert et al. 2000). This cell cycle reentry defect correlates closely with a defect in the cell-cycle-dependent induction of classic E2F-responsive genes (Humbert et al. 2000). We hypothesized that the expression of Arf might also be altered in the $E 2 f 3^{-/-}$MEFs if $A r f$ is a genuine E2F-target gene. To test this idea, we used serum deprivation to arrest early-passage wild-type and $E 2 f 3^{-/-}$
MEFs in $\mathrm{G}_{0} / \mathrm{G}_{1}$ and harvested cells at various time points after serum re-addition to assess the state of cell cycle reentry (through analysis of incorporated tritiated thymidine) and gene expression (by western blotting). Consistent with our previous studies, the E2f3 ${ }^{-/-}$MEFs showed a dramatic reduction in the level of mitogen-induced DNA replication (Fig. 2A) and the expression of cyclin A, a classic E2F-responsive gene (Fig. 2B). As previously described (Sherr and Weber 2000), p19 ${ }^{\text {Arf }}$ was present at very low levels in the arrested wild-type cells, and its expression was not induced during the cell cycle reentry (Fig. 2B). In contrast, p19 $9^{\text {Arf }}$ was dramatically up-regulated in the $E 2 \mathrm{f3}^{-/-}$MEFs in both the arrested and the mitogen-treated cells (Fig. 2B). Quantitative RT-PCR shows that this reflects an increase in the level of Arf mRNA (data not shown). Thus, E2f3 inactivation alters the transcription of $A r f$ in the opposite manner from its effect on classic E2F-responsive genes.

The predominant function of $\mathrm{p} 19^{\mathrm{Arf}}$ is to inhibit the ubiquitin ligase $\mathrm{mdm} 2$ and consequently trigger the stabilization and activation of p53. Consistent with this scheme, the E2f3 mutant MEFs displayed the classic hallmarks of $\mathrm{p} 53$ activation: There was an increase in the levels of p53 that was phosphorylated on Ser-15 and a dramatic up-regulation in the levels of the cdk inhibitor

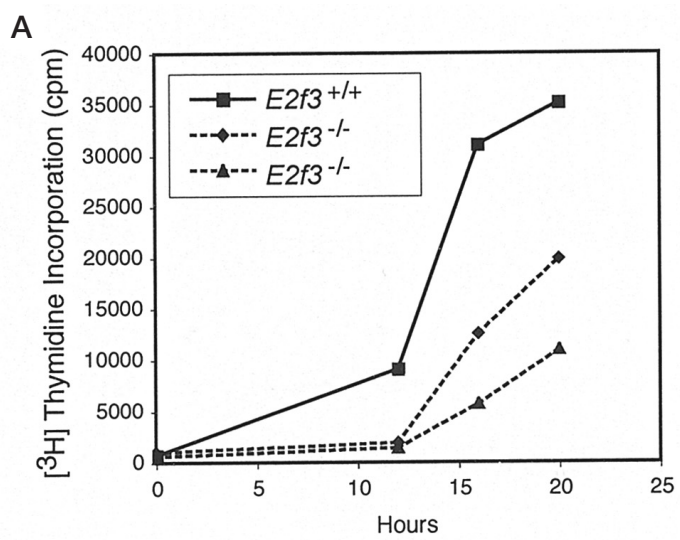

B

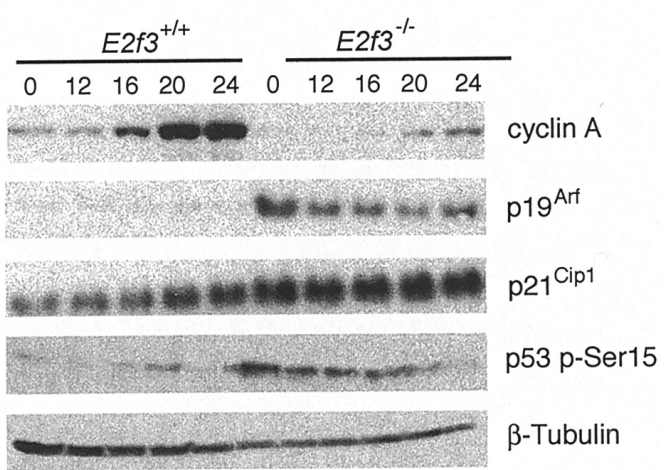

Figure 2. $E 2 f 3^{--}$MEFs are defective in cell cycle reentry, due to activation of $\mathrm{p} 19^{\text {Arf }}$ and $\mathrm{p} 53$. (A) Wild-type MEFs (solid line) or $E 2 \mathrm{f3}^{-1-}$ MEFs (dotted lines) were synchronized by serum starvation, and cell cycle reentry was monitored by $\left[{ }^{3} \mathrm{H}\right]$ thymidine incorporation into newly synthesized DNA at the indicated times. $(B)$ Total protein extracts were prepared at the indicated times after serum stimulation and subjected to western blotting for cyclin A, p19 ${ }^{\text {Arf }}, \mathrm{p} 21^{\text {Cip1 }}$, phospho-p53(Ser15), or $\beta$-Tubulin. 
p21 $1^{\text {Cip1 }}$, a known transcriptional target of p53 (Fig. 2B). Thus, E2f3 inactivation leads to the induction of $A r f$ and the activation of $\mathrm{p} 53$. It is well documented that changes in the levels of p19 ${ }^{\mathrm{Arf}}, \mathrm{p} 53$, and $\mathrm{p} 21^{\mathrm{Cip} 1}$ can have a major effect on the properties of MEFs, particularly in their ability to undergo mitogen-induced cell cycle reentry (Sherr and Weber 2000). Consistent with these observations, we found that the loss of either Arf or $p 53 \mathrm{com}$ pletely suppressed the cell cycle reentry defects of the $E 23^{-1-}$ MEFs, including the defect in the expression of classic E2F-responsive genes (Aslanian et al. 2004). This genetic rescue could arise in two ways. The loss of $\operatorname{Arf}$ (or p53) could confer a proliferative advantage on the cells that outweighs or overrides the proliferative disadvantage that results from E2f3 loss. Alternatively, it could reflect the existence of a linear pathway in which E2F3 is required to repress $A r f$, and therefore prevents activation of p53 and $\mathrm{p} 21^{\text {Cip } 1 .}$

To determine whether E2F family members are directly involved in the regulation of $A r f$ in unstressed wildtype cells, we conducted ChIP assays (Fig. 3). As a positive control, we first examined E2F binding to a classic E2F-responsive promoter, $p 107$. In an asynchronous population of MEFs, we were able to detect E2F1, 2, 3, and 4 in association with the p107 promoter (Fig. 3). This is consistent with the documented role of both repressive (E2F4) and activating (E2F1, 2, and 3A) E2Fs in controlling the cell-cycle-dependent expression of this classic target gene (Trimarchi and Lees 2002). Remarkably, we observed a dramatically different pattern of E2F binding at the Arf promoter: It was occupied by E2F3 but not other E2F family members. Notably, this ChIP signal was observed using an antibody that recognizes both E2F3A and E2F3B, but not an antibody that is specific for E2F3A (Fig. 3). This raises the possibility that $A r f$ is specifically targeted by the E2F3B isoform.

The transcription of classic E2F-responsive genes is activated in late $\mathrm{G}_{1}$ because of a switch in promoter occupancy from repressive to activating E2F complexes. Given the differential E2F-binding properties of Arf versus classic E2F-responsive genes in asynchronous cells, we wanted to establish how Arf was regulated at distinct

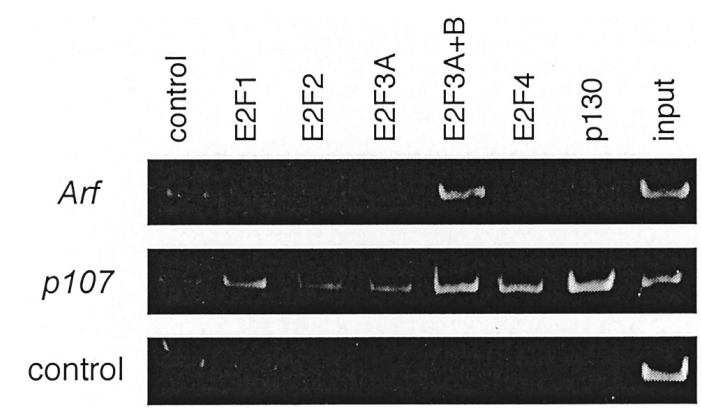

Figure 3. The Arf promoter is specifically bound by E2F3. ChIP analysis was performed on asynchronously growing wild-type $\mathrm{MEFs}$, and binding of E2F and pocket protein complexes to the Arf and 107 promoters was monitored. Whereas the classic E2F-responsive promoter, p107, is bound by all E2Fs, E2F3 is the only species detected at the Arf promoter.

cell cycle stages. To address this issue, we used serum deprivation/restimulation assays to isolate cells at various stages of arrest or cell cycle reentry. As with our previous studies (see Fig. 2, for example), the cells initiate DNA synthesis $16-20$ hours after serum stimulation. As expected, we found that the 107 promoter, a classic E2F target, was bound by the repressive E2F4-p130 complex in arrested cells (0h; Fig. 4). In the enriched S-phase population, the E2F4/p130 signal was reduced and the activating E2Fs, E2F1, and E2F3A, clearly associated with the p107 promoter (20h; Fig. 4). In striking contrast, E2F3 was the only E2F that was bound to the Arf promoter in arrested MEFs. Moreover, this E2F3 binding was maintained during cell cycle reentry and we did not observe recruitment of any other E2Fs to the Arf promoter (Fig. 4). This analysis clearly shows that normal proliferative signals encountered during cell cycle entry are insufficient to drive E2F activation of Arf. Rather, Arf expression is kept low throughout the cell cycle, and this correlates with the binding of E2F3. In the $E 2 f 3^{-/}$MEFs, the derepression of $A r f$ occurs without any detectable binding of the activating E2Fs (data not shown). Thus, we conclude that E2F3 contributes to the transcription repression of $\operatorname{Arf}$ in unstressed cells.

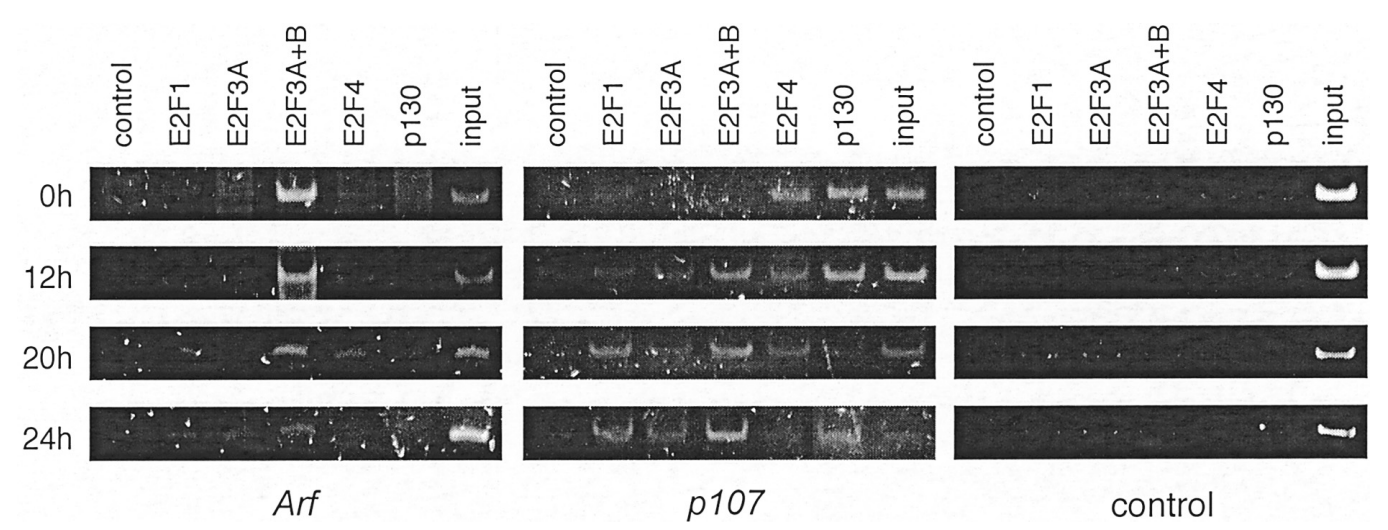

Figure 4. The Arf promoter is not activated by E2F during cell cycle reentry. Wild-type MEFs were synchronized by serum withdrawal, stimulated with $10 \%$ serum for the indicated times, and then subjected to ChIP analysis. Whereas the activating E2Fs are recruited to the $p 107$ promoter, the Arf promoter is bound only by E2F3 throughout cell cycle reentry. 


\section{Activating E2Fs Are Involved in the Oncogenic Activation of $A r f$}

The experiments above establish a direct role for E2F3 in repression of Arf in unstressed cells. Given these observations, we wanted to determine whether/how the E2F regulation of $A r f$ changes when the transcription of this gene is induced by oncogenic challenge. To address this question, we examined the effect of overexpressing the adenoviral oncoprotein, E1A. This is a potent oncogene that promotes both proliferation and apoptosis. E1A acts, at least in part, by sequestering the pocket proteins and relieving the transcriptional inhibition of the endogenous activating E2Fs (Ben-Israel and Kleinberger 2002). We infected early-passage wild-type MEFs with either control or E1A-expressing retroviruses and then used ChIP to assess E2F binding to either the Arf or $p 107$ promoters (Fig. 5). Infection with the control virus did not alter the spectrum of E2F complexes of these cells: Arf was still specifically occupied by E2F3 while p107 was bound by E2F1, 2, 3, and 4. E1A expression had a fairly subtle effect on the spectrum of E2F complexes that were bound to the $p 107$ promoter. This included a modest reduction in the binding of the repressive E2F, E2F4, and a subtle in-

A

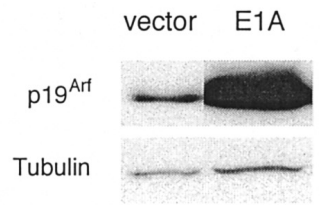

B
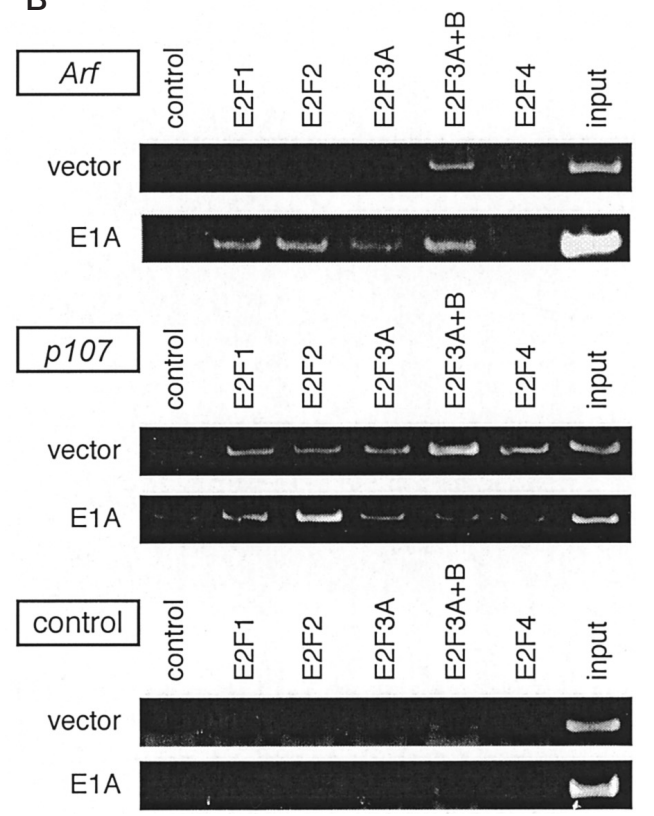

Figure 5. The activating E2Fs bind to the Arf promoter in response to oncogenic stress. Wild-type MEFs were infected with retrovirus overexpressing $\mathrm{E} 1 \mathrm{~A}$, or an empty virus (vector) and subjected to western $(A)$ or ChIP $(B)$ analyses with the indicated antisera. MEFs expressing E1A exhibit dramatically increased levels of p19 ${ }^{\text {Arf }}$, coincident with recruitment of activating E2Fs (E2F1, E2F2, and E2F3A) to the Arf promoter. crease in the binding of the activating E2Fs, E2F1 and 2, that are consistent with E1A's documented ability to promote the expression of classic E2F target genes (Fig. 5). Importantly, E1A had a much more profound effect on Arf. There was a dramatic recruitment of the activating E2Fs, E2F1, 2, and 3A, to the Arf promoter, and this correlated with a huge increase in the expression of $\mathrm{p} 19^{\text {Arf }}$ (Fig. 5). Given these findings, we conclude that the endogenous activating E2Fs participate in the activation of Arf, and consequently, the tumor surveillance network engaged by the E1A oncogene.

\section{DISCUSSION}

Tumor development is highly dependent on the deregulation of two regulatory networks, the $\mathrm{p} 16^{\text {Ink4a }}$ cycD/cdk4-pRB-E2F pathway that controls cellular proliferation and the p19 ${ }^{\text {Arf }}$-mdm2-p53 axis that mediates the tumor surveillance response (Sherr 2001). Our data reveal a direct connection between these two networks: E2F plays a key role in controlling the transcription of the Arf tumor suppressor in both normal and tumor cells.

Through a combination of genetic and biochemical evidence, we have shown that E2F3 contributes directly to the transcriptional repression of $A r f$ in unstressed cells. The E2f3 locus is known to encode two distinct isoforms, E2F3A and E2F3B (He et al. 2000; Leone et al. 2000). E2F3A is expressed in proliferating cells, and it is widely accepted to be an activating E2F (Trimarchi and Lees 2002; Dimova and Dyson 2005). In contrast, E2F3B is expressed in both quiescent and proliferating cells and, based solely on this expression pattern, was proposed to be a transcriptional repressor (Leone et al. 2000). Our ChIP data implicate E2F3B in the transcriptional repression of Arf. Specifically, an antibody that is specific for E2F3A yielded a clear ChIP signal at our classic E2F-responsive gene, p107, but not at Arf, whereas an antibody that recognizes the common carboxyl terminus of both E2F3A and E2F3B detects E2F3 binding to both $\mathrm{Arf}$ and $p 107$ promoters. These observations do not rule out the possibility that E2F3A contributes to the transcription repression of Arf, but they suggest that $\mathrm{E} 2 \mathrm{~F} 3 \mathrm{~B}$ is the major player. This represents the first functional evidence that the E2F3B protein is a repressor of transcription.

Numerous E2F family members have been classified as repressor E2Fs (Trimarchi and Lees 2002; Dimova and Dyson 2005). Of these, E2F4 and 5 are the best characterized and the most closely related to E2F3B. E2F4 and 5 repress transcription of classic E2F-responsive genes in $\mathrm{G}_{0} / \mathrm{G}_{1}$ by virtue of their association with the pocket proteins, and their associated histone-modifying enzymes. This repression is relieved as cells enter the cell cycle, when phosphorylation of the pocket protein by CDK activity leads to a disruption of the E2F-pocket protein complex. E2F3B is known to associate specifically with $\mathrm{pRB}$ in the $\mathrm{G}_{1}$ phase (Leone et al. 2000). However, we were not able to detect $\mathrm{pRB}$, or any other pocket protein, binding to the $A r f$ promoter (Figs. 3 and 4; data not shown), raising the possibility that $\mathrm{E} 2 \mathrm{~F} 3 \mathrm{~B}$ enforces repression of 
Arf in a pRB-independent manner. Notably, the binding of E2F3B to Arf persists during cell cycle entry even though CDK activity is high, unlike the E2F4-p130 complex, which is lost from the $p 107$ promoter during cell cycle entry (Fig. 3). This difference supports the idea that the repressive E2F3B complex is refractory to CDK activity and therefore does not contain $\mathrm{pRB}$. At least two other known repressors of Arf, Bmi-1 and CBX7, are members of the Polycomb group of transcriptional repressors (Jacobs et al. 1999; Gil et al. 2004). Since it has been shown previously that another repressive E2F, E2F6, acts in association with Polycomb proteins (Trimarchi et al. 2001; Ogawa et al. 2002; Attwooll et al. 2005), it is feasible that E2F3B may cooperate with Bmi1 and/or CBX7 to engage repression of Arf.

In addition to their well-known role in regulation of cell cycle entry and DNA synthesis, E2F proteins have also been implicated in regulation of genes involved in the apoptotic response. However, there is considerable debate about whether these pro-apoptotic genes are genuine targets in vivo and, more contentiously, which of the E2F family members are capable of inducing apoptosis. A number of studies argue that the induction of apoptosis is a specific property of E2F1 and not other E2F family members (DeGregori et al. 1997; Leone et al. 2001; Denchi and Helin 2005). This specificity could be due, at least in part, to the presence of a domain unique to E2F1 that binds $\mathrm{pRB}$ and specifically regulates its apoptotic activity (Dick and Dyson 2003). However, other studies show that the activating E2Fs can all induce apoptosis when they are ectopically expressed (Vigo et al. 1999; Baudino et al. 2003). Clearly, these studies do not rule out the possibility that E2F2 and E2F3A might promote apoptosis indirectly by activating E2F1, a known E2F-responsive gene. Unfortunately, various mouse models give conflicting answers to this issue. For example, it was recently reported that the apoptosis arising in an E2f3a transgenic mouse model occurs in an E2f1-dependent manner (Denchi and Helin 2005). However, other models clearly show that the absence of $E 2 f 1$, or indeed both $E 2 f 1$ and $E 2 f 2$, does not alter the ability of cells to induce Arf or undergo apoptosis in response to oncogene activation (Palmero et al. 2002; Baudino et al. 2003). Our data clearly show that E2F1, 2, and 3A all associate with the Arf promoter when this gene is induced by the presence of E1A (Fig. 5 ). Notably, this validates a role for the endogenous E2Fs, as opposed to overexpressed E2Fs, in the oncogenic response. On the basis of these observations, we conclude that $\operatorname{Arf}$ is a genuine E2F target and that all three activating E2Fs cooperate in the activation of this pro-apoptotic gene. This redundancy, combined with the need to achieve a certain total level of activating E2Fs, could explain the previous conflicting observations that one or more of the activating E2Fs can be either essential or dispensable for the activation of $\mathrm{Arf}$ and/or apoptosis in different settings.

Unlike most E2F target genes, Arf expression is not induced as cells reenter the cell cycle from a quiescent state (Fig. 2). We show here that this is likely due to the failure of activating E2Fs to bind to the Arf promoter during cell cycle entry, as well as the persistence of the repressive E2F3 complex (Fig. 4). However, many questions remain regarding this unique regulation of Arf. How is E2F3 specifically targeted to Arf? What prevents activation of Arf by other E2Fs during cell cycle entry? And how is oncogenic stress sensed, ultimately leading to activating E2F binding to Arf? To address this last question: It is possible that the switch from repression to activation requires a modification of either the E2F3-repressive complex or the activating E2F species, which only occurs in conditions of oncogene-induced hyperproliferation. Alternatively, the overall level of E2F activity may be the key determinant. During normal proliferation, perhaps the level of E2F activity is only sufficient to bind and activate the promoters of classic E2F targets. In contrast, the expression of E1A could yield a supra-physiological level of activating E2Fs that allows binding and activation of both classic E2F-responsive genes and additional targets like Arf. Importantly, this "supra-activation" model could account for the activity of other cellular oncogenes, including Ras, c-Myc, and Abl. These factors all possess the dual ability to promote cellular proliferation, ultimately by promoting the release of the activating E2Fs, and also activate the Arf tumor surveillance network. It is tempting to speculate that some, or all, of these factors would activate $A r f$ as an unintended consequence of their need to engage E2F (Fig. 6). At least in the case of ras, it is clear that another transcription factor, Dmp1, contributes to

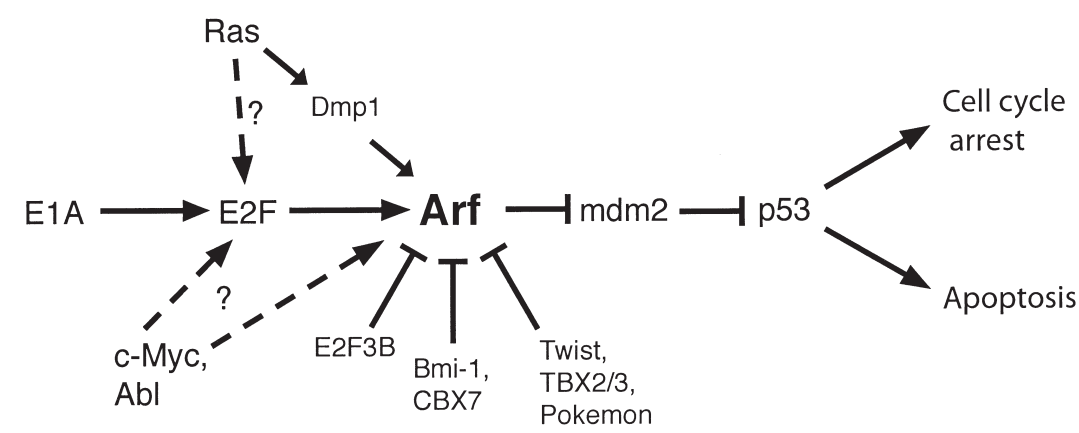

Figure 6. The Arf-p53 pathway is subject to complex regulation. Arf is known to be regulated, both positively and negatively, by numerous factors (solid lines). This study shows that E1A-induced activation of Arf occurs via E2F. It remains possible that signaling to Arf from other oncogenes (Ras, c-Myc, Abl) may occur through E2F (dotted lines). 
Arf activation (Fig. 6) (Sreeramaneni et al. 2005). However, the levels of the activating E2Fs could act as a global sensor that would distinguish inappropriate from normal proliferation and mobilize $A r f$.

\section{ACKNOWLEDGMENTS}

J.A.L. is a Daniel K. Ludwig Scholar, and P.I. was supported by a Ludwig predoctoral fellowship. This work was supported by a grant from the National Institutes of Health (PO1-CA42063) to J.A.L.

\section{REFERENCES}

Adams M.R., Sears R., Nuckolls F., Leone G., and Nevins J.R. 2000. Complex transcriptional regulatory mechanisms control expression of the E2F3 locus. Mol. Cell. Biol. 20: 3633.

Aslanian A., Iaquinta P.J., Verona R., and Lees J.A. 2004. Repression of the Arf tumor suppressor by E2F3 is required for normal cell cycle kinetics. Genes Dev. 18: 1413.

Attwooll C., Oddi S., Cartwright P., Prosperini E., Agger K., Steensgaard P., Wagener C., Sardet C., Moroni M.C., and Helin K. 2005. A novel repressive E2F6 complex containing the polycomb group protein, EPC1, that interacts with EZH2 in a proliferation-specific manner. J. Biol. Chem. 280: 1199.

Baudino T.A., Maclean K.H., Brennan J., Parganas E., Yang C., Aslanian A., Lees J.A., Sherr C.J., Roussel M.F., and Cleveland J.L. 2003. Myc-mediated proliferation and lymphomagenesis, but not apoptosis, are compromised by E2f1 loss. Mol. Cell 11: 905.

Ben-Israel H. and Kleinberger T. 2002. Adenovirus and cell cycle control. Front. Biosci. 7: d1369.

Berkovich E., Lamed Y., and Ginsberg D. 2003. E2F and Ras synergize in transcriptionally activating p14ARF expression. Cell Cycle 2: 127.

Cam H. and Dynlacht B.D. 2003. Emerging roles for E2F: Beyond the G1/S transition and DNA replication. Cancer Cell 3: 311.

Cam H., Balciunaite E., Blais A., Spektor A., Scarpulla R.C., Young R., Kluger Y., and Dynlacht B.D. 2004. A common set of gene regulatory networks links metabolism and growth inhibition. Mol. Cell 16: 399.

Cartwright P., Muller H., Wagener C., Holm K., and Helin K. 1998. E2F-6: A novel member of the E2F family is an inhibitor of E2F-dependent transcription. Oncogene 17: 611.

Christensen J., Cloos P., Toftegaard U., Klinkenberg D., Bracken A.P., Trinh E., Heeran M., Di Stefano L., and Helin K. 2005. Characterization of E2F8, a novel E2F-like cell-cycle regulated repressor of E2F-activated transcription. $\mathrm{Nu}$ cleic Acids Res. 33: 5458.

de Bruin A., Maiti B., Jakoi L., Timmers C., Buerki R., and Leone G. 2003. Identification and characterization of E2F7, a novel mammalian E2F family member capable of blocking cellular proliferation. J. Biol. Chem. 278: 42041.

DeGregori J., Leone G., Miron A., Jakoi L., and Nevins J.R. 1997. Distinct roles for E2F proteins in cell growth control and apoptosis. Proc. Natl. Acad. Sci. 94: 7245.

Denchi E.L. and Helin K. 2005. E2F1 is crucial for E2F-dependent apoptosis. EMBO Rep. 6: 661.

de Stanchina E., McCurrach M.E., Zindy F., Shieh S.Y., Ferbeyre G., Samuelson A.V., Prives C., Roussel M.F., Sherr C.J., and Lowe S.W. 1998. E1A signaling to p53 involves the p19(ARF) tumor suppressor. Genes Dev. 12: 2434.

Dick F.A. and Dyson N. 2003. pRB contains an E2F1-specific binding domain that allows E2F1-induced apoptosis to be regulated separately from other E2F activities. Mol. Cell 12: 639.

Dimova D.K. and Dyson N.J. 2005. The E2F transcriptional network: Old acquaintances with new faces. Oncogene 24: 2810.

Di Stefano L., Jensen M.R., and Helin K. 2003. E2F7, a novel E2F featuring DP-independent repression of a subset of E2Fregulated genes. EMBO J. 22: 6289.
Gaubatz S., Lees J.A., Lindeman G.J., and Livingston D.M. 2001. E2F4 is exported from the nucleus in a CRM1-dependent manner. Mol. Cell. Biol. 21: 1384.

Gaubatz S., Lindeman G.J., Ishida S., Jakoi L., Nevins J.R., Livingston D.M., and Rempel R.E. 2000. E2F4 and E2F5 play an essential role in pocket protein-mediated G1 control. Mol. Cell 6: 729 .

Gil J., Bernard D., Martinez D., and Beach D. 2004. Polycomb CBX7 has a unifying role in cellular lifespan. Nat. Cell Biol. 6: 67.

Hanahan D. and Weinberg R.A. 2000. The hallmarks of cancer. Cell 100: 57.

He Y., Armanious M.K., Thomas M.J., and Cress W.D. 2000. Identification of E2F-3B, an alternative form of E2F-3 lacking a conserved N-terminal region. Oncogene 19: 3422.

Hiebert S.W., Packham G., Strom D.K., Haffner R., Oren M., Zambetti G., and Cleveland J.L. 1995. E2F-1: DP-1 induces $\mathrm{p} 53$ and overrides survival factors to trigger apoptosis. Mol. Cell. Biol. 15: 6864.

Hsieh J.K., Fredersdorf S., Kouzarides T., Martin K., and Lu X. 1997. E2F1-induced apoptosis requires DNA binding but not transactivation and is inhibited by the retinoblastoma protein through direct interaction. Genes Dev. 11: 1840.

Humbert P.O., Verona R., Trimarchi J.M., Rogers C., Dandapani S., and Lees J.A. 2000. E2f3 is critical for normal cellular proliferation. Genes Dev. 14: 690.

Jacobs J.J., Kieboom K., Marino S., DePinho R.A., and van Lohuizen M. 1999. The oncogene and Polycomb-group gene bmi-1 regulates cell proliferation and senescence through the ink4a locus. Nature 397: 164.

Johnson D.G., Cress W.D., Jakoi L., and Nevins J.R. 1994. Oncogenic capacity of the E2F1 gene. Proc. Natl. Acad. Sci. 91: 12823 .

Landsberg R.L., Sero J.E., Danielian P.S., Yuan T.L., Lee E.Y., and Lees J.A. 2003. The role of E2F4 in adipogenesis is independent of its cell cycle regulatory activity. Proc. Natl. Acad. Sci. 100: 2456.

Lees J.A., Saito M., Vidal M., Valentine M., Look T., Harlow E., Dyson N., and Helin K. 1993. The retinoblastoma protein binds to a family of E2F transcription factors. Mol. Cell. Biol. 13: 7813 .

Leone G., Nuckolls F., Ishida S., Adams M., Sears R., Jakoi L., Miron A., and Nevins J.R. 2000. Identification of a novel $\mathrm{E} 2 \mathrm{~F} 3$ product suggests a mechanism for determining specificity of repression by Rb proteins. Mol. Cell. Biol. 20: 3626.

Leone G., Sears R., Huang E., Rempel R., Nuckolls F., Park C.H., Giangrande P., Wu L., Saavedra H.I., Field S.J., Thompson M.A., Yang H., Fujiwara Y., Greenberg M.E., Orkin S., Smith C., and Nevins J.R. 2001. Myc requires distinct E2F activities to induce S phase and apoptosis. Mol. Cell 8: 105.

Lukas J., Petersen B.O., Holm K., Bartek J., and Helin K. 1996. Deregulated expression of E2F family members induces Sphase entry and overcomes p16INK4A-mediated growth suppression. Mol. Cell. Biol. 16: 1047.

Maiti B., Li J., de Bruin A., Gordon F., Timmers C., Opavsky R., Patil K., Tuttle J., Cleghorn W., and Leone G. 2005. Cloning and characterization of mouse E2F8, a novel mammalian E2F family member capable of blocking cellular proliferation. $J$. Biol. Chem. 280: 18211.

McCaffrey J., Yamasaki L., Dyson N.J., Harlow E., and Griep A.E. 1999. Disruption of retinoblastoma protein family function by human papillomavirus type $16 \mathrm{E} 7$ oncoprotein inhibits lens development in part through E2F-1. Mol. Cell. Biol. 19: 6458.

Moberg K., Starz M.A., and Lees J.A. 1996. E2F-4 switches from p130 to p107 and pRB in response to cell cycle reentry. Mol. Cell. Biol. 16: 1436.

Morkel M., Wenkel J., Bannister A.J., Kouzarides T., and Hagemeier C. 1997. An E2F-like repressor of transcription. Nature 390: 567.

Morris E.J. and Dyson N.J. 2001. Retinoblastoma protein partners. Adv. Cancer Res. 82: 1.

Ogawa H., Ishiguro K., Gaubatz S., Livingston D.M., and Nakatani Y. 2002. A complex with chromatin modifiers that 
occupies E2F- and Myc-responsive genes in G0 cells. Science 296: 1132

Palmero I., Murga M., Zubiaga A., and Serrano M. 2002. Activation of ARF by oncogenic stress in mouse fibroblasts is independent of E2F1 and E2F2. Oncogene 21: 2939.

Pan H., Yin C., Dyson N.J., Harlow E., Yamasaki L., and Van Dyke T. 1998. Key roles for E2F1 in signaling p53-dependent apoptosis and in cell division within developing tumors. Mol. Cell 2: 283.

Parisi T., Pollice A., Di Cristofano A., Calabro V., and La Mantia G. 2002. Transcriptional regulation of the human tumor suppressor p14(ARF) by E2F1, E2F2, E2F3, and Sp1-like factors. Biochem. Biophys. Res. Commun. 291: 1138.

Phillips A.C. and Vousden K.H. 2001. E2F-1 induced apoptosis. Apoptosis 6: 173.

Phillips A.C., Bates S., Ryan K.M., Helin K., and Vousden K.H. 1997. Induction of DNA synthesis and apoptosis are separable functions of E2F-1. Genes Dev. 11: 1853.

Phillips A.C., Ernst M.K., Bates S., Rice N.R., and Vousden K.H. 1999. E2F-1 potentiates cell death by blocking antiapoptotic signaling pathways. Mol. Cell 4: 771.

Qin X.Q., Livingston D.M., Kaelin W.G., Jr., and Adams P.D. 1994. Deregulated transcription factor E2F-1 expression leads to S-phase entry and p53-mediated apoptosis. Proc. Natl. Acad. Sci. 91: 10918.

Ren B., Cam H., Takahashi Y., Volkert T., Terragni J., Young R.A., and Dynlacht B.D. 2002. E2F integrates cell cycle progression with DNA repair, replication, and $\mathrm{G}(2) / \mathrm{M}$ checkpoints. Genes Dev. 16: 245.

Serrano M., Lee H., Chin L., Cordon-Cardo C., Beach D., and DePinho R.A. 1996. Role of the INK4a locus in tumor suppression and cell mortality. Cell 85: 27.

Sherr C.J. 1996. Cancer cell cycles. Science 274: 1672.

. 2001. The INK4a/ARF network in tumour suppression. Nat. Rev. Mol. Cell Biol. 2: 731

Sherr C.J. and Weber J.D. 2000. The ARF/p53 pathway. Curr. Opin. Genet. Dev. 10: 94.
Sreeramaneni R., Chaudhry A., McMahon M., Sherr C.J., and Inoue K. 2005. Ras-Raf-Arf signaling critically depends on the Dmp1 transcription factor. Mol. Cell. Biol. 25: 220.

Takahashi Y., Rayman J.B., and Dynlacht B.D. 2000. Analysis of promoter binding by the E2F and $\mathrm{pRB}$ families in vivo: Distinct E2F proteins mediate activation and repression. Genes Dev. 14: 804

Trimarchi J.M. and Lees J.A. 2002. Sibling rivalry in the E2F family. Nat. Rev. Mol. Cell Biol. 3: 11.

Trimarchi J.M., Fairchild B., Wen J., and Lees J.A. 2001. The E2F6 transcription factor is a component of the mammalian Bmil-containing polycomb complex. Proc. Natl. Acad. Sci. 98: 1519 .

Trimarchi J.M., Fairchild B., Verona R., Moberg K., Andon N., and Lees J.A. 1998. E2F-6, a member of the E2F family that can behave as a transcriptional repressor. Proc. Natl. Acad. Sci. 95: 2850.

Verona R., Moberg K., Estes S., Starz M., Vernon J.P., and Lees J.A. 1997. E2F activity is regulated by cell cycle-dependent changes in subcellular localization. Mol. Cell. Biol. 17: 7268.

Vigo E., Muller H., Prosperini E., Hateboer G., Cartwright P., Moroni M.C., and Helin K. 1999. CDC25A phosphatase is a target of E2F and is required for efficient E2F-induced S phase. Mol. Cell. Biol. 19: 6379.

Wu X. and Levine A.J. 1994. p53 and E2F-1 cooperate to mediate apoptosis. Proc. Natl. Acad. Sci. 91: 3602.

Yamasaki L., Bronson R., Williams B.O., Dyson N.J., Harlow E., and Jacks T. 1998. Loss of E2F-1 reduces tumorigenesis and extends the lifespan of Rb1(+/-)mice. Nat. Genet. 18: 360 .

Ziebold U., Lee E.Y., Bronson R.T., and Lees J.A. 2003. E2F3 loss has opposing effects on different $\mathrm{pRB}$-deficient tumors, resulting in suppression of pituitary tumors but metastasis of medullary thyroid carcinomas. Mol. Cell. Biol. 23: 6542 .

Zindy F., Williams R.T., Baudino T.A., Rehg J.E., Skapek S.X., Cleveland J.L., Roussel M.F., and Sherr C.J. 2003. Arf tumor suppressor promoter monitors latent oncogenic signals in vivo. Proc. Natl. Acad. Sci. 100: 15930. 


\section{$\$_{\text {SSH\& }}^{\infty} \mathrm{CS}$ Cold Spring Harbor Symposia SYMPOSIA on Quantitative Biology}

\section{Regulation of the Arf/p53 Tumor Surveillance Network by E2F}

P.J. IAQUINTA, A. ASLANIAN and J.A. LEES

Cold Spring Harb Symp Quant Biol 2005 70: 309-316

Access the most recent version at doi:10.1101/sqb.2005.70.050

References This article cites 61 articles, 33 of which can be accessed free at: http://symposium.cshlp.org/content/70/309.full.html\#ref-list-1

License

Email Alerting Receive free email alerts when new articles cite this article - sign up in Service the box at the top right corner of the article or click here. 